\title{
Equilibration of Photogenerated Charge Carriers in Plas- monic Core@Shell Nanoparticles
}

\author{
Matteo Parente $^{\dagger, \ddagger}$, Sassan Sheikholeslami ${ }^{\S}$, Gururaj V. Naik ${ }^{\|}$, Jennifer A. Dionne ${ }^{\ddagger}$, Andrea Baldi ${ }^{\dagger, \ddagger}{ }^{\dagger}$ \\ † DIFFER - Dutch Institute for Fundamental Energy Research, De Zaale 20, 5612 AJ Eindhoven, The Netherlands \\ ‡ ICMS - Institute for Complex Molecular Systems, Eindhoven University of Technology, P.O. Box 513, 5600 BM Eindhoven, The Netherlands \\ § Pacific Biosciences, Menlo Park, CA 94025, USA
}

|| Department of Electrical \& Computer Engineering, Rice University, 610o Main St. MS-378 Houston TX 77005, USA

$¥$ Department of Materials Science and Engineering, Stanford University, Stanford, CA 94305, USA

ABSTRACT: Noble metal nanoparticles support localized surface plasmon resonances (LSPRs), which are light-driven oscillations of free
electrons. Thanks to their strong dependence on the metal's electron density, these resonances can be used to optically probe the equilibra-
tion of photogenerated charge carriers at metal/semiconductor interfaces, a process of paramount importance in energy conversion and
sensing applications. In practice, however, it is often difficult to obtain quantitative insight from the observed plasmonic effects, as the spectral
position, intensity, and linewidth of LSPRs can be influenced by different competing contributions, such as particle size distribution, surface
oxidation, and changes in the dielectric environment. Here, we develop a nearly monodisperse synthesis of $\mathrm{Ag}_{\mathrm{T}} \mathrm{TiO}_{2}$ and $\mathrm{Au}_{\mathrm{TiO}} \mathrm{TiO}_{2}$
core@shell nanoparticles and measure their plasmon resonance shifts during UV irradiation and charge equilibration in the dark. We show
that the observed optical response can be fully accounted for by an accumulation of photogenerated electrons in the TiO $\mathrm{T}_{2}$ shells and that
charge transfer to the metal cores is negligible. Our results challenge the established understanding of charge equilibration in hybrid met-
al@semiconductor nanostructures.

\section{INTRODUCTION}

Noble metal nanoparticles strongly absorb and scatter light, thanks to so-called localized surface plasmon resonances (LSPRs) ${ }^{1,2}$. These resonances are very sensitive to variations in the particle's surrounding medium and in their charge density, making them ideally suited as optical probes of chemical reactions and energy conversion processes ${ }^{3,4,5,6}$. For example, plasmon resonances have been used to detect $\mathrm{CO}$ and $\mathrm{H}_{2}$ oxidation on $\mathrm{Pt}^{7}$, adsorption of pure gases on $\mathrm{Ag}^{8}$, and $\mathrm{H}_{2}$ intercalation in $\mathrm{Pd}^{9}$. In biochemistry, LSPRs have been used to detect the hybridization of DNA strands ${ }^{10}$, conformational changes in proteins ${ }^{11}$, and the presence of unlabelled proteins in solution ${ }^{12}$. Furthermore, reactions involving charge transfer to and from metal nanoparticles can be optically followed thanks to the spectral dependence of the LSPR on the charge density of the metal ${ }^{13,14}$. For example, scattering spectroscopy has been used to follow the oxidation of ascorbic acid on single gold nanoparticles $^{15}$. Recently, metal nanoparticles have been shown to enhance exciton separation in metal/semiconductor heterostructures ${ }^{16-18}$. Such charge separation has been followed spectroscopically as a blue shift in the plasmon resonance of Agand Au-decorated $\mathrm{TiO}_{2}$ nanostructures ${ }^{19-22}$. However, several questions remain on the interpretation of the observed resonance shifts during charge equilibration in these nanoscale heterostructures. First, the observed resonance shifts often lead to the prediction of large increases in the charge density of the metal nanoparticle (of the order of several percentages) ${ }^{21,23,24}$ which, in the absence of any electrical double layer screening from counterions in solu$\operatorname{tion}^{15,25}$, would result in extremely high electric fields. Second, the magnitude of the plasmon resonance shift is strongly dependent on the size of the nanoparticles ${ }^{3,14}$, leading to large uncertainties in samples with a wide size distribution ${ }^{21,26}$. Third, the spectral shifts in the plasmon resonance wavelength are typically accompanied by variations in the peak's full width at half maximum (FWHM) and in its intensity, which are often difficult to attribute to a single effect $^{25,27,28}$.

Here, we tackle these questions by studying the equilibration of photogenerated charge carriers in colloidal suspensions of nearly monodisperse $\mathrm{Ag} @ \mathrm{TiO}_{2}$ and $\mathrm{Au} @ \mathrm{TiO}_{2}$ core@shell nanoparticles. The narrow size distribution of our nanoparticles afforded by our two-step synthetic procedure, allows us to derive quantitative conclusions on the mechanism of plasmon resonance shift. In particular, we find that electron storage in the metallic cores is negligible and that the observed LSPR shifts can be fully accounted for by the irreversible reduction of surface oxide at the metal/ $\mathrm{TiO}_{2}$ interface and by the reversible accumulation of electrons in the $\mathrm{TiO}_{2}$ shell.

\section{EXPERIMENTAL SECTION}

All the chemicals have been used as received, without any further purification. $\mathrm{AgNO}_{3}$ (99.9999\%), $\mathrm{HAuCl}_{4}$ trihydrate ( $\left.\geq 99.9 \%\right)$, sodium citrate dihydrate ( $\geq 99 \%)$, tannic acid (ACS grade reagent), TTIP (99.999\%), and HPC $\left(\mathrm{M}_{\mathrm{w}} \sim 80000, \mathrm{M}_{\mathrm{n}} \sim 10000\right)$ were purchased from Sigma Aldrich. TTIP was stored and handled in a 
glovebox to prevent any hydrolysis due to air moisture. Dehydrated $\mathrm{Na}_{2} \mathrm{SO}_{4}$ (>99\%) was purchased from Fluka. Ethanol (Seccosolv dried, $\max 0.01 \% \mathrm{H}_{2} \mathrm{O}$ ) was purchased from Merck Millipore. Ultrapure water $18.2 \mathrm{M} \Omega \mathrm{cm}$ was obtained from mQ Integral Water Purification System by Merck Millipore.

UV-Vis extinction spectra are acquired with a Perkin Elmer Lambda 1050 spectrophotometer equipped with 3D WB detector module. Scanning electron microscopy images are obtained using a Zeiss Sigma field emission SEM operating at $10 \mathrm{kV}$. Scanning transmission electron microscopy (STEM) images are measured on an FEI Verios 460 SEM equipped with a STEM retractable detector.

Synthesis of Ag nanoparticles. Nearly monodisperse Ag nanoparticles were prepared by adapting a literature protocol ${ }^{29}$. In a typical synthesis, a $20 \mathrm{~mL}$ aqueous solution made of $6.8 \mathrm{mM}$ of sodium citrate dihydrate and $100 \mu \mathrm{M}$ tannic acid is heated to $65^{\circ} \mathrm{C}$. An 80 $\mathrm{mL}$ acqueous solution of $0.74 \mathrm{mM} \mathrm{AgNO}_{3}$ is also heated to $65^{\circ} \mathrm{C}$. The two hot solutions are mixed together under vigorous stirring and the temperature is raised till boiling. The mixture is kept boiling without reflux for 30 minutes then cooled down to room temperature. The Ag nanoparticles are purified by centrifuging $25 \mathrm{~mL}$ of the final solution at $7900 \mathrm{xg}$ for 30 minutes. The supernatant is removed and the nanoparticles are redispersed in $25 \mathrm{~mL}$ of ultrapure water. The solution is centrifuged again at $7900 \mathrm{xg}$ for 30 minutes, the supernatant is removed and the nanoparticles are redispersed in $2 \mathrm{~mL}$ of ultrapure water. Finally, the solution is filtered through $0.45 \mu \mathrm{m}$ and $0.2 \mu \mathrm{m}$ filters (Acrodisk) and the nanoparticles are immediately used for the $\mathrm{TiO}_{2}$ shell synthesis. The final solution of cleaned Ag nanoparticles has an optical density of $>100$ at the silver plasmon resonance and it is stable in the dark at $4{ }^{\circ} \mathrm{C}$ for about 1 week. The average size of the Ag nanoparticles is $35.6 \pm 7.2 \mathrm{~nm}$ in diameter, with a LSPR peak centered around $407 \mathrm{~nm}$ in water.

Synthesis of Au nanoparticles. Nearly monodisperse Au nanoparticles were prepared by following a procedure reported in the literature $^{30}$. Gold nanoparticles are purified using the same procedure developed for the silver ones. In this case, the final optical density of the solution is $\sim 5$ at the gold LSPR. The average diameter of the $\mathrm{Au}$ nanoparticles is $34.7 \pm 4.5 \mathrm{~nm}$, with an LSPR peak centered at $\sim 527 \mathrm{~nm}$.

Synthesis of $\mathrm{Ag} @ \mathrm{TiO}_{2}$ nanoparticles. The $\mathrm{TiO}_{2}$ shell is formed in a sol-gel reaction in which titanium isopropoxide (TTIP) is hydrolysed on the surface of the purified Ag nanoparticles in the presence of water and hydroxypropyl cellulose (HPC) as a stabilizing agent $^{31}$. In a typical synthesis, a $2 \mathrm{~mL}$ solution of HPC-stabilized Ag nanoparticles with an optical density $\mathrm{OD}=20$ at the plasmon resonance wavelength $\left(\lambda_{\mathrm{LSPR}}=407 \mathrm{~nm}\right)$ is obtained by dispersing the proper amount of purified $\mathrm{Ag}$ nanoparticles into an aqueous solution of $1 \mu \mathrm{M}$ HPC. A $10 \mathrm{~mL} 3.4 \mathrm{mM}$ ethanolic solution of TTIP is added to the HPC-stabilized Ag nanoparticles with a syringe pump, at a rate of $0.5 \mathrm{~mL} / \mathrm{min}$ at room temperature, under vigorous stirring. The final product consists of a mixture of $\mathrm{Ag} @ \mathrm{TiO}_{2}$ and $\mathrm{TiO}_{2}$ nanoparticles. This final solution is boiled for 100 minutes under reflux and then let cool down to room temperature. $15 \mathrm{~mL}$ are transferred into ten $1.5-\mathrm{mL}$ centrifuge vials and centrifuged at $600 \mathrm{xg}$ for 90 minutes. The precipitate is redispersed to an optical density of 0.3 using $0.17 \mu \mathrm{M}$ ethanolic solution of
HPC. An excess of anhydrous $\mathrm{Na}_{2} \mathrm{SO}_{4}$ is added to the $\mathrm{Ag} @ \mathrm{TiO}_{2}$ NPs to minimize water content and the solution is then stored at 4 ${ }^{\circ} \mathrm{C}$ in the dark. The average thickness of the $\mathrm{TiO}_{2}$ shell is $\sim 8 \mathrm{~nm}$, and the LSPR peak of the $\mathrm{Ag} @ \mathrm{TiO}_{2}$ nanoparticles is centered around $446 \mathrm{~nm}$.

Synthesis of $\mathbf{A u} @ \mathrm{TiO}_{2}$ nanoparticles. Synthesis of $\mathrm{Au} @ \mathrm{TiO}_{2}$ nanoparticles has been carried out by adapting the one developed for Ag@ $\mathrm{TiO}_{2}$ for a lower starting OD. In a typical synthesis, a $2 \mathrm{~mL}$ solution of HPC-stabilized Au nanoparticles with an optical density $\mathrm{OD}=5$ at the plasmon resonance wavelength $\left(\lambda_{\mathrm{LSPR}}=527 \mathrm{~nm}\right)$ is obtained by dispersing the proper amount of purified Au nanoparticles into an aqueous solution of $1 \mu \mathrm{M}$ HPC. A $10 \mathrm{~mL} 0.85 \mathrm{mM}$ ethanolic solution of TTIP is added to the HPC-stabilized Au nanoparticles with a syringe pump at a rate of $0.5 \mathrm{~mL} / \mathrm{min}$ at room temperature under vigorous stirring. The solution is then boiled for 100 minutes under reflux. The final product consists of a mixture of $\mathrm{Au} @ \mathrm{TiO}_{2} \mathrm{NPs}$ and $\mathrm{TiO}_{2}$ nanoparticles. The $\mathrm{Au} @ \mathrm{TiO}_{2}$ nanoparticles are purified with the same procedure used for Ag@ $\mathrm{TiO}_{2}$. The average thickness of the $\mathrm{TiO}_{2}$ shell is $\sim 12 \mathrm{~nm}$, and the LSPR peak of the $\mathrm{Au} @ \mathrm{TiO}_{2}$ nanoparticles is centered around $550 \mathrm{~nm}$.

Charge equilibration measurements. In each experiment, we use $4 \mathrm{~mL}$ of $\mathrm{Ag} @ \mathrm{TiO}_{2}$ or $\mathrm{Au} @ \mathrm{TiO}_{2}$ suspensions in a $0.17 \mu \mathrm{M}$ ethanolic solution of HPC with an optical density of 0.3 at the respective LSPRs. The solution is initially purged for 20 minutes with $\mathrm{N}_{2}$, to minimize the presence of oxygen, and the $\mathrm{N}_{2}$ purging is continued throughout the whole experiment. The solution undergoes five cycles of UV light exposure for 10 minutes, followed by 30 minutes of charge equilibration in the dark. We use $254 \mathrm{~nm}$ UV light from an Oriel-Newport calibration lamp (model 6034) driven by $18 \mathrm{~mA}$ $\mathrm{A} / \mathrm{C}$ current and filtered with a $350 \mathrm{~nm}$ short-pass filter. Extinction spectra are continuously acquired every 5 seconds using a low power white light source and an OceanOptics HR4000 CG-UVNIR spectrophotometer (see Figure S1 for the experimental setup).

\section{RESULTS AND DISCUSSION}

We first developed two-step syntheses of Ag@ $\mathrm{TiO}_{2}$ and $\mathrm{Au} @ \mathrm{TiO}_{2}$ core@shell nanoparticles that allow to accurately control the size of the metal cores as well as the thickness of the shell. Silver nanoparticles were synthesized by adapting a previously published method ${ }^{29}$ based on the reduction of silver nitrate by sodium citrate, in the presence of tannic acid (TA). The role of tannic acid is to chelate $\mathrm{Ag}^{+}$ions, with high $\mathrm{Ag}^{+} / \mathrm{TA}$ ratios leading to fast nucleation of metallic silver clusters and smaller final sizes of the nanoparticles. The final nanoparticle suspension is stabilized by the citrate anion, which acts as both reducing and capping agent. Citrate-stabilized gold nanoparticles were synthesized following a literature meth$\mathrm{od}^{30}$. As-synthesized $\mathrm{Ag}$ and $\mathrm{Au}$ nanoparticles are purified by centrifugation to remove excess citrate, which can hinder the $\mathrm{TiO}_{2}$ shell formation. The purified nanoparticles are then coated with a $\mathrm{TiO}_{2}$ shell by the sol-gel hydrolysis of an ethanolic solution of titanium tetraisopropoxide (TTIP) in presence of water (final ethanol:water ratio of 5:1) and hydroxypropyl cellulose (HPC) as a surfactant ${ }^{31,32}$. The thickness of the $\mathrm{TiO}_{2}$ shell can be modulated by the rate of dropwise addition of the TTIP solution. The Ag@ $\mathrm{TiO}_{2}$ and $\mathrm{Au} @ \mathrm{TiO}_{2}$ nanoparticles are finally centrifuged and redispersed 
in an ethanolic HPC solution to minimize water content and remove excess $\mathrm{TiO}_{2}$ nanoparticles, which are a by-product of the solgel synthesis. Further details on the synthetic procedures can be found in the Materials and Methods section.

Scanning electron microscope (SEM) images of the $\mathrm{Ag}$ and $\mathrm{Au}$ cores and scanning transmission electron microscope (STEM) images of the $\mathrm{Ag} @ \mathrm{TiO}_{2}$ and $\mathrm{Au} @ \mathrm{TiO}_{2}$ nanoparticles are shown in Figure $1 \mathrm{a}$ and Figure $\mathrm{1b}$, respectively. For the $\mathrm{Ag}$ and Au cores we obtain nearly monodisperse particles with an average diameter of $\sim 35 \mathrm{~nm}$ and LSPRs of aqueous suspensions centered at 407 and $527 \mathrm{~nm}$, respectively. Upon formation of a $\mathrm{TiO}_{2}$ shell, the LSPRs red-shift by $39 \mathrm{~nm}\left(\mathrm{Ag} @ \mathrm{TiO}_{2}\right)$ and $23 \mathrm{~nm}\left(\mathrm{Au} @ \mathrm{TiO}_{2}\right)$, due to the higher refractive index of $\mathrm{TiO}_{2}$ with respect to the solvent, and the signature of the $\mathrm{TiO}_{2}$ band-gap becomes clearly visible as a strong absorption in the UV part of the spectrum (Figure 1c).

Charge equilibration in $\mathrm{Ag} @ \mathrm{TiO}_{2}$ and $\mathrm{Au} @ \mathrm{TiO}_{2}$ nanoparticle suspensions is studied by irradiating them with UV light under inert $\left(\mathrm{O}_{2}\right.$ free $)$ atmosphere. Ultraviolet irradiation causes electronhole pair formation in the $\mathrm{TiO}_{2}$ shell. The photogenerated holes are quickly scavenged away by the ethanol. In the absence of $\mathrm{O}_{2}$, which is an efficient electron scavenger, electrons are therefore accumulated in the $\mathrm{TiO}_{2}$ shell. We study the equilibration of these photogenerated electrons by continuously measuring the extinction spectra of the core@shell nanoparticle suspensions upon repeated cycles of UV irradiation and equilibration in the dark. In a typical experiment, $4 \mathrm{~mL}$ of solution with an optical density (OD) of 0.3 at the LSPR wavelength are sealed in a quartz cuvette. To remove any trace of oxygen in the liquid, the ethanolic solution is purged for 20 minutes with $\mathrm{N}_{2}$ gas before UV exposure, and the solution is kept under continuous nitrogen purging during the entire measurement. The solution is then subjected to 5 consecutive cycles of UV exposure for 10 minutes followed by 30 minutes in the dark. During the course of the entire experiment, extinction spectra are acquired every 5 seconds using a low-power white light source and a fiber optical spectrometer (further details in the Materials and Methods section and Figure $\mathrm{S} 1$ ).

In Figure 2, we show the raw UV-Vis spectra for both $\mathrm{Ag} @ \mathrm{TiO}_{2}$ and $\mathrm{Au} @ \mathrm{TiO}_{2}$ nanoparticles before the measurement, after 10 minutes of UV exposure, and after 30 minutes of charge equilibration in the dark. Notably, for both $\mathrm{Ag} @ \mathrm{TiO}_{2}$ and $\mathrm{Au} @ \mathrm{TiO}_{2}$ nanoparticles, after 10 minutes of UV exposure the LSPR peaks are blueshifted, the FWHMs decrease and the peak intensities increase. These changes are partially reversible after 30 minutes in the dark.
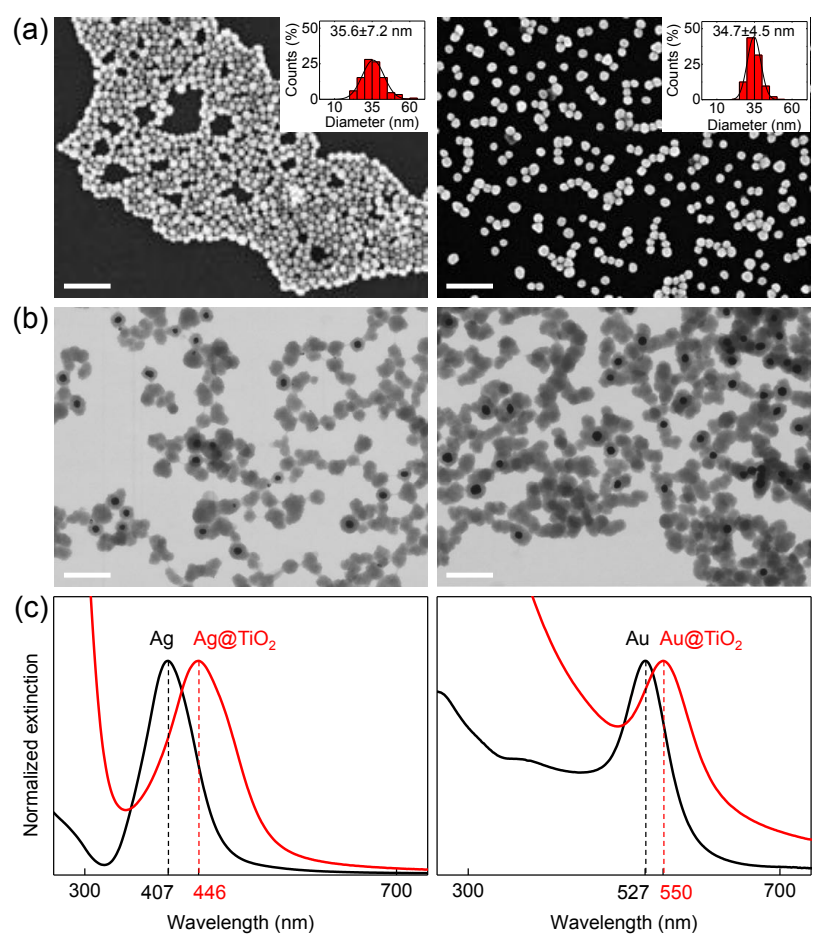

Figure 1. (a) SEM images of (left) Ag and (right) Au nanoparticles on silicon substrates. The insets show the measured size distributions for $\mathrm{Ag}(35.6 \pm 7.2 \mathrm{~nm}$, average of 471 particles) and $\mathrm{Au}(34.7 \pm 4.5 \mathrm{~nm}$, average of 280 particles). (b) STEM images of HPC-stabilized (left) $\mathrm{Ag} @ \mathrm{TiO}_{2}$ and (right) $\mathrm{Au} @ \mathrm{TiO}_{2}$ nanoparticles dispersed on ultrathin carbon membranes. The measured average shell thicknesses for the $\mathrm{Ag} @ \mathrm{TiO}_{2}$ and $\mathrm{Au} @ \mathrm{TiO}_{2}$ nanoparticles are $8 \mathrm{~nm}$ and $12 \mathrm{~nm}$, respectively. All scale bars are $200 \mathrm{~nm}$. (c) Normalized extinction spectra of (left) $\mathrm{Ag}$ and $\mathrm{Ag} @ \mathrm{TiO}_{2}$ and (right) $\mathrm{Au}$ and $\mathrm{Au} @ \mathrm{TiO}_{2}$ nanoparticle suspensions.

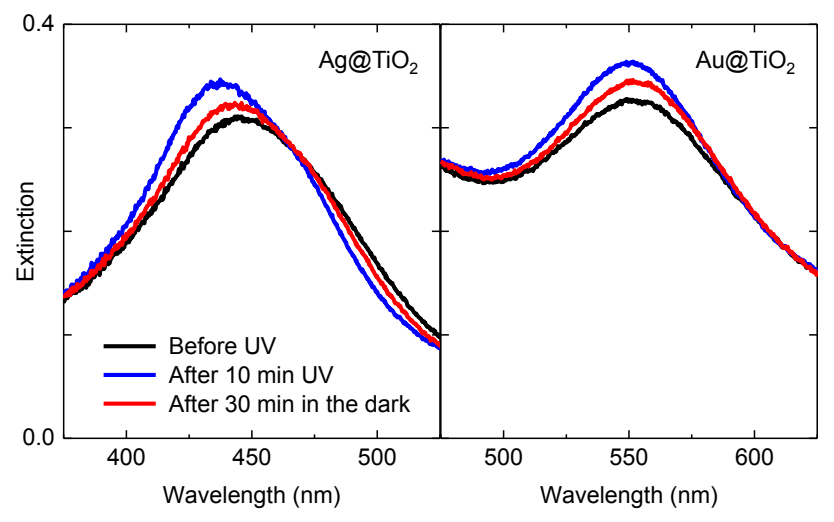

Figure 2. Extinction spectra of (left) $\mathrm{Ag} @ \mathrm{TiO}_{2}$ and (right) $\mathrm{Au} @ \mathrm{TiO}_{2}$ nanoparticle suspensions (black) before and (blue) after the first UV exposure for 10 minutes and (red) after equilibration in the dark for 30 minutes.

Figure 3a shows the time evolution of the LSPR peak wavelength for $\mathrm{Ag} @ \mathrm{TiO}_{2}$ and $\mathrm{Au} @ \mathrm{TiO}_{2}$ over 5 consecutive cycles of UV irradiation and dark equilibration. While $\mathrm{Au} @ \mathrm{TiO}_{2}$ nanoparticles show a reversible blueshift, the plasmon resonance of $\mathrm{Ag} @ \mathrm{TiO}_{2}$ nanoparticles only partially recovers its initial spectral position upon cycling, with a cumulative irreversible blueshift over the 5 cycles of $\sim 4 \mathrm{~nm}$. The observed difference between particles with 
silver and (more noble) gold cores allows us to attribute such irreversible blueshift to the reduction of the native $\mathrm{Ag}_{2} \mathrm{O}$ at the $\mathrm{Ag} / \mathrm{TiO}_{2}$ interface. It is in fact well known that the reduction of the native surface oxide in silver nanoparticles leads to a blueshift and an increase in the extinction intensity of their plasmon resonanc$\mathrm{es}^{33}$, in agreement with our experimental observation (see also Figure 2). To estimate the thickness of the reduced silver oxide, we use Mie theory to calculate the LSPR peak position of $\mathrm{Ag} @ \mathrm{Ag}_{2} \mathrm{O} @ \mathrm{TiO}_{2}$ core@shell@shell multilayered spheres with varying $\mathrm{Ag}_{2} \mathrm{O}$ thicknesses ${ }^{34}$. We find that an irreversible blueshift of $\sim 4 \mathrm{~nm}$ corresponds to the reduction of an interface $\mathrm{Ag}_{2} \mathrm{O}$ layer with a thickness of $\sim 0.2 \mathrm{~nm}$ (see also SI 2), roughly corresponding to a monolayer of oxygen atoms at the silver surface ${ }^{35}$. Interestingly, the largest irreversible reduction of silver oxide occurs during the first cycle of UV exposure, while subsequent cycles are similar in the magnitude of their reversible LSPR shifts. Another experimental evidence supporting the claim that the irreversible LSPR shift is due to the reduction of an interface $\mathrm{Ag}_{2} \mathrm{O}$ layer, is the fact that the initial LSPR peak position can be recovered at the end of the experiment upon exposure of the solution to air (see Figure S3). The absence of an irreversible component for $\mathrm{Au} @ \mathrm{TiO}_{2}$ nanoparticles suggests that their surface is not oxidized, in agreement with both experiments and theoretical predictions for $\mathrm{Au}$ nanoparticles larger than $4 \mathrm{~nm}$ in diameter ${ }^{36}$.
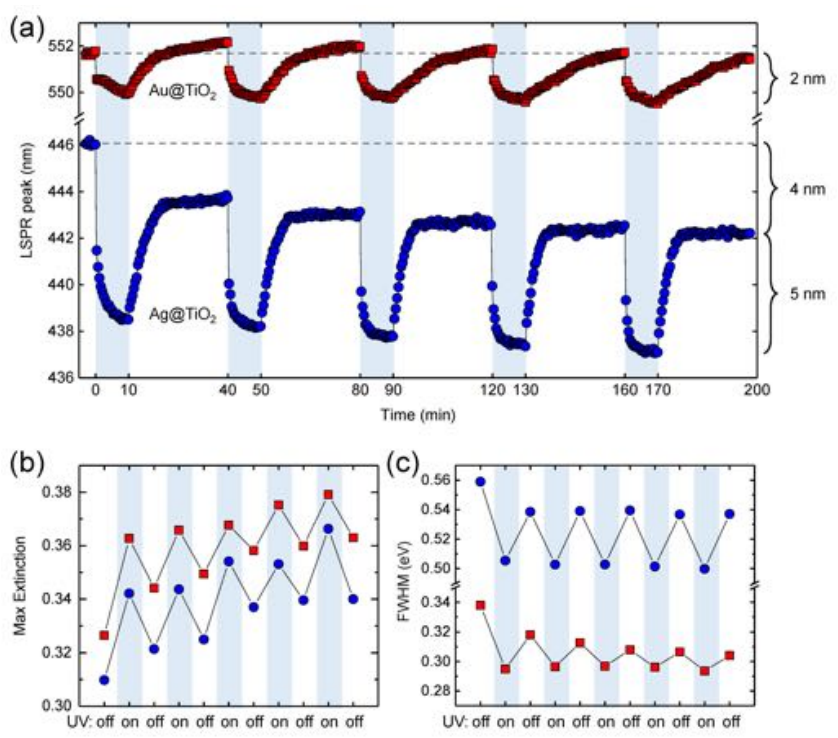

Figure 3. (a) Measured spectral positions of the LSPR peak maxima for $\mathrm{Ag} @ \mathrm{TiO}_{2}$ (blue dots) and $\mathrm{Au} @ \mathrm{TiO}_{2}$ (red squares) nanoparticle solutions, during 5 consecutive cycles of UV exposure and dark equilibration under $\mathrm{N}_{2}$ purging. The pale blue regions highlight the intervals of UV exposure. After $5 \mathrm{UV}$ irradiation cycles an irreversible blueshift of $\sim 4 \mathrm{~nm}$ is present for $\mathrm{Ag} @ \mathrm{TiO}_{2}$ nanoparticles, as well as a reversible blueshift of $\sim 5 \mathrm{~nm}$. For Au@ $\mathrm{TiO}_{2}$ nanoparticles, we observe only a reversible blueshift of $\sim 2 \mathrm{~nm}$. Steady-state values of $(b)$ the extinction maximum and (c) the FWHM of the resonance peaks for $\mathrm{Ag} @ \mathrm{TiO}_{2}$ (blue dots) and $\mathrm{Au} @ \mathrm{TiO}_{2}$ (red squares) nanoparticle solutions at the end of the UV irradiation (on) and after equilibration in the dark (off). UV irradiation is always accompanied by an increase in the extinction maximum and a decrease in the FWHM of the resonance peak.

Besides the irreversible effect due to the reduction of silver oxide, Figure 3a also shows reversible LSPR shifts for both $\mathrm{Ag} @ \mathrm{TiO}_{2}$ $\left(\Delta \lambda_{\mathrm{LSPR}} \approx 5 \mathrm{~nm}\right)$ and $\mathrm{Au} @ \mathrm{TiO}_{2}\left(\Delta \lambda_{\mathrm{LSPR}} \approx 2 \mathrm{~nm}\right)$ nanoparticle solutions. A generally accepted interpretation in the literature explains the reversible LSPR blue-shifts upon UV irradiation of metal/semiconductor hybrid nanostructures with an accumulation of electrons in the metal, while holes are scavenged away by the solvent ${ }^{18,21,23}$. In the Drude approximation, the frequency of a plasmon resonance is in fact proportional to the square root of the charge density in the metal ${ }^{14}$. Here, however, we show that this interpretation is incorrect and that the observed resonance shifts are inconsistent with an accumulation of electrons in the cores. Using Mie theory, we can calculate the charge density increase necessary to induce a $5 \mathrm{~nm}$ blue shift in the LSPR of Ag@ $@ \mathrm{TiO}_{2}$ nanoparticles. In our calculations, we use a dielectric function of titania that we experimentally measure by ellipsometry on a sol-gel spin-coated $\mathrm{TiO}_{2}$ thin film (see Figure S4). For the metal cores, we fit a Drude model to the dielectric function of silver ${ }^{37}$ and vary the plasma frequency to account for an increased charge density (see Figure S5). Assuming that the effective number of electrons per silver atom contributing to its optical properties is approximately one $^{38}$, we find that we can reproduce the observed LSPR shift with a charge density increase of $\sim 2.7 \%$, corresponding to the storage of $\sim 3.5 \times 10^{4}$ electrons per $\mathrm{Ag} @ \mathrm{TiO}_{2}$ nanoparticle. The accumulation of electrons in a coated metal nanoparticle leads to an increase of its Fermi energy given by ${ }^{39}$

$$
\Delta \mathrm{E}_{\mathrm{F}}=\frac{(2 \mathrm{z}-1) \mathrm{e}}{8 \pi \varepsilon_{0}(\mathrm{r}+\mathrm{d})}\left(\frac{\mathrm{d}}{\varepsilon_{\mathrm{d}} \mathrm{r}}+\frac{1}{\varepsilon_{\mathrm{r}}}\right)
$$

where $\mathrm{z}$ is the number of excess electrons accumulated in the metallic core $\left(z=3.5 \times 10^{4}\right)$, e is the elementary charge $\left(e=1.6 \times 10^{-19} \mathrm{C}\right)$, $\mathrm{r}$ is the radius of the metal core $(\mathrm{r}=17.5 \mathrm{~nm}), \mathrm{d}$ and $\varepsilon_{\mathrm{d}}$ are the thickness and the relative static permittivity of the shell, respectively $\left(\mathrm{d}=8 \mathrm{~nm}, \mathcal{E}_{\mathrm{d}, \mathrm{TiO} 2}=26\right)^{40,41}$, and $\mathcal{E}_{\mathrm{r}}$ is the relative static permittivity of the solvent $\left(\varepsilon_{\mathrm{r}, \mathrm{EtOH}}=24.3\right)^{42}$. The accumulation of $\sim 3.5 \times 10^{4}$ electrons in a single $\mathrm{Ag} @ \mathrm{TiO}_{2}$ nanoparticle would therefore lead to an impossible Fermi energy increase in the silver core of $\sim 116 \mathrm{eV}$, a value which is much larger than the work function of the metal itself $\left(\Phi_{\mathrm{Ag}}=4.26 \mathrm{eV}\right)^{43}$. A similar unphysical result is obtained if we hypothesize that the holes scavenged by the ethanol, instead of being transported away into the solvent, are localized at the $\mathrm{TiO}_{2} / \mathrm{EtOH}$ interface. Such charge distribution would in fact minimize the electrostatic energy of the system. In this scenario, we can treat our core@shell nanoparticles as a spherical capacitor whose plates are separated by a distance corresponding to the $\mathrm{TiO}_{2}$ thickness $(8 \mathrm{~nm})$. Using the electrostatic equation for a spherical capacitor and the relative static permittivity of $\mathrm{TiO}_{2}$ leads to a predicted electric field across the shell of $\sim 4 \mathrm{~V} / \mathrm{nm}$, which is one to two orders of magnitude higher than the reported breakdown voltage of $\mathrm{TiO}_{2}{ }^{41,44}$. Finally, according to the Drude model and Mie theory, the LSPR blue-shift due to charge accumulation in metal nanoparticles must always be accompanied by a widening of the FWHM and a lowering of the extinction cross-section ${ }^{25}$ (see Figure S5). This is opposite to what we observe experimentally, as shown in Figure 3b and Figure 3c. Upon UV irradiation of both $\mathrm{Ag@} \mathrm{TiO}_{2}$ and $\mathrm{Au} @ \mathrm{TiO}_{2}$ nanoparticles, in fact, we observe a narrowing of the FWHM (see also details in Figure S6) and an increase in the extinction cross-section at the LSPR peak. These changes are partially reversible upon charge equilibration in the dark. The above considerations, together with the slow kinetics of the LSPR shifts (of the order of minutes), strongly suggest that charge accumulation in the metal cores can be neglected and that a different mechanism must be at play. 
To understand the physical origin of the reversible LSPR shifts, we first consider the observed difference in magnitude between the results obtained for $\mathrm{Ag} @ \mathrm{TiO}_{2}\left(\Delta \lambda_{\mathrm{LSPR}} \approx 5 \mathrm{~nm}\right)$ and $\mathrm{Au} @ \mathrm{TiO}_{2}$ $\left(\Delta \lambda_{\text {LSPR }} \approx 2 \mathrm{~nm}\right)$. It is known that the LSPR of silver nanoparticles is more spectrally sensitive to variations in the refractive index of the surrounding environment than the LSPR of gold nanoparticles. This can be easily modeled using Mie theory (see Figure S7) and it is already evident by comparing the LSPR shifts observed when coating bare $\mathrm{Ag}$ and $\mathrm{Au}$ nanoparticles with a similar shell thickness of $\mathrm{TiO}_{2}$ (Figure 1c). In the absence of any significant charge accumulation effects in the metal cores, the only mechanism that can explain the observed LSPR shifts in our nanoparticles is therefore a UV-induced change in the dielectric properties of their $\mathrm{TiO}_{2}$ shells. In the following we will show that the reversible LSPR shifts of our core@shell nanoparticles can be fully accounted for by the accumulation and depletion of electrons in $\mathrm{TiO}_{2}$.

The accumulation of electrons in $\mathrm{TiO}_{2}$ is typically accompanied by an increase in its optical absorption in the visible ${ }^{21,45-47}$, due to electrons trapped at $\mathrm{Ti}^{4+}$ sites $^{21}$. To verify that electron storage also occurs in our colloidally prepared titania, we studied the photoresponse of $\mathrm{TiO}_{2}$ nanoparticles without metallic cores. The absence of any plasmon resonance in their extinction spectrum allows us to observe optical changes due to effects occurring only in $\mathrm{TiO}_{2}$. In Figure 4a we show optical extinction spectra of a colloidal suspension of $\mathrm{TiO}_{2}$ nanoparticles before and after 10 minutes of UV irradiation under inert atmosphere. We observe a large extinction increase across the visible part of the spectrum, consistent with an accumulation of electrons in the $\mathrm{TiO}_{2}$ nanoparticles ${ }^{21}$.

The UV-induced accumulation of trapped electrons in $\mathrm{TiO}_{2}$ is known to strongly increase its hydrophilicity ${ }^{4-50}$. Our $\mathrm{TiO}_{2}$ is prepared by a sol-gel method followed by reflux, which typically results in amorphous and highly porous shells ${ }^{51,52}$ (see also Figure S8). A larger wettability of such $\mathrm{TiO}_{2}$ shells would therefore induce an uptake of solvent in its pores and a consequent thickness increase ${ }^{49}$. The swelling of $\mathrm{TiO}_{2}$ leads to a reduction of its density and therefore a decrease in its refractive index according to the LorentzLorenz equation $^{53-55}$

$$
\frac{n_{e f f}^{2}-1}{n_{e f f}^{2}+2}=A \rho^{B}
$$

where $\mathrm{n}_{\text {eff }}$ is the effective refractive index, $\rho$ is the density, and A and $\mathrm{B}$ are fitted parameters specific to $\mathrm{TiO}_{2}{ }^{56}$. Using this equation we performed Mie theory calculations of the extinction cross-section of both $\mathrm{Ag} @ \mathrm{TiO}_{2}$ and $\mathrm{Au} @ \mathrm{TiO}_{2}$ nanoparticles with variable shell thicknesses. We find that the observed reversible blueshifts for both $\mathrm{Ag} @ \mathrm{TiO}_{2}$ and $\mathrm{Au} @ \mathrm{TiO}_{2}$ can be quantitatively explained by a thickness increase in their shells of $\sim 0.2 \mathrm{~nm}$ (see details in the Figure S9). Such a small variation is entirely reasonable for soft, colloidally synthesized $\mathrm{TiO}_{2}$ shells (see Figure S8) and further highlights the extreme spectral sensitivity of our plasmonic core@shell nanoparticles. Furthermore, the UV-induced increase in hydrophilicity for $\mathrm{TiO}_{2}$ has been shown to be reversible upon storage in the dark, with slow discharge kinetics ${ }^{50}$. This is in agreement with our observed rates of LSPR redshift (Figure 3a), which we attribute to the slow scavenging of the accumulated electrons in $\mathrm{TiO}_{2}$ by traces of oxidized reactants, such as oxidized EtOH or traces of $\mathrm{H}_{2} \mathrm{O}$ and $\mathrm{O}_{2}$.
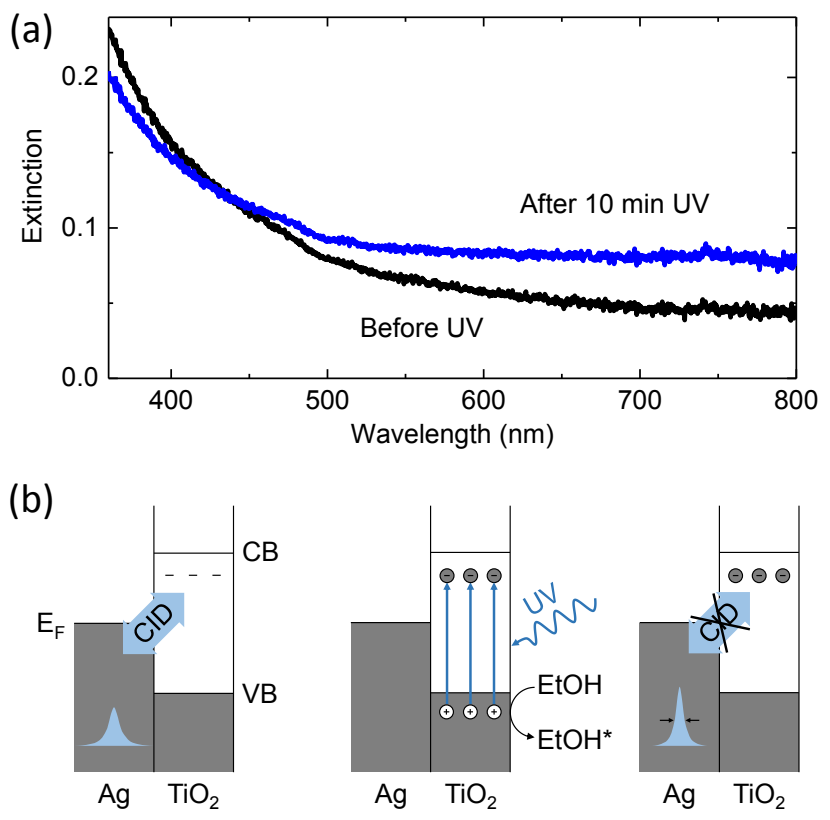

Figure 4. (a) Extinction spectra of a suspension of colloidal $\mathrm{TiO}_{2}$ nanoparticles in ethanol, (black curve) before and (blue curve) after exposure to UV irradiation for 10 minutes. The solution is constantly purged with $\mathrm{N}_{2}$ gas to minimize the content of $\mathrm{O}_{2}$. (b) Schematic energy diagram of the $\mathrm{Ag} / \mathrm{TiO}_{2}$ interface (left) before, (center) during, and (right) after UV irradiation.

As we have shown in Figure $3 b$ and Figure 3c, the UV-induced blueshift of the LSPR is always accompanied by an increase of the extinction maximum and a decrease of the FWHM. These effects cannot be due to the presence of pure $\mathrm{TiO}_{2}$ nanoparticles as byproducts in our core@shell nanoparticle suspensions (see Figure S10) and clearly indicate a reduced damping of the plasmon resonance as electrons are accumulated in the $\mathrm{TiO}_{2}$ shell. It is easy to demonstrate with Mie theory that a reduced damping in a core@shell metal@semiconductor nanoparticle could be explained by a reduction in the extinction coefficient of the semiconducting shell. Titanium dioxide is however almost completely transparent in the visible, with an extinction coefficient close to zero for energies between 1-3.5 eV (see SI 4). Furthermore, a decrease of the extinction coefficient of $\mathrm{TiO}_{2}$ is incompatible with the observed increase in extinction shown in Figure 4 a. We can gain an insight into the physical mechanism of the observed decrease in plasmon damping by considering the electronic band structure at the met$\mathrm{al} / \mathrm{TiO}_{2}$ interface, as schematically depicted in Figure $4 \mathrm{~b}$. Due to the amorphous and defective nature of the sol gel $\mathrm{TiO}_{2}$, there are empty acceptor levels below the conduction band ${ }^{57}$. Before UV irradiation, these empty levels can contribute to the plasmon broadening through chemical interface damping (CID) $)^{58,59}$. CID is sometimes also referred to as the "direct" mechanism of charge transfer from a metal nanoparticle to an acceptor electronic level at its surface ${ }^{60}$, and it is known to significantly broaden the LSPR of coated nanoparticles ${ }^{27,28,61-63}$. Upon UV irradiation in inert atmosphere, EtOH scavenges the photogenerated holes and electrons accumulate in the midgap states. These electrons prevent direct charge transfer from the metal nanoparticles to $\mathrm{TiO}_{2}$ and effectively quench the CID mechanism. In Figure $3 c$ we show that, upon UV irradiation, the linewidth of the plasmon resonances of $\mathrm{Ag} @ \mathrm{TiO}_{2}$ and $\mathrm{Au} @ \mathrm{TiO}_{2}$ decrease by a few tens of meV. Interestingly, a CID damping parameter of the order of tens of $\mathrm{meV}$ has also been re- 
cently reported for Au nanorods coated with thiol ligands ${ }^{28}$. Furthermore, it has been suggested that a change in chemical interface damping could also affect the spectral position of the plasmon resonance, due to the dependence of the LSPR frequency on the damping parameter $\gamma^{28}$. Such dependence could in principle qualitatively explain the observed LSPR shifts (Figure 3a), without invoking any swelling of the $\mathrm{TiO}_{2}$ shells. However, for silver and gold, characterized in the Drude approximation by plasma frequencies of the order of $\sim 10 \mathrm{eV}$, a variation in the damping parameter of the order of tens of $\mathrm{meV}$ has a negligible effect on the spectral position of the LSPR (see Figure S11).

\section{CONCLUSIONS}

In summary, we have characterized the plasmonic response of nearly monodisperse core@shell Ag@ $\mathrm{TiO}_{2}$ and $\mathrm{Au} @ \mathrm{TiO}_{2}$ nanoparticles, under UV irradiation in inert atmosphere. We find that the UV-induced changes in their plasmon resonance peak position, intensity and linewidth can be fully explained by the accumulation of electrons in the $\mathrm{TiO}_{2}$ shells, without invoking any charge transfer to the metal cores. The photogenerated electrons in $\mathrm{TiO}_{2}$ lead to a swelling of the shell and to the quenching of chemical interface damping. In the case of $\mathrm{Ag} @ \mathrm{TiO}_{2}$, some of the photogenerated electrons are also used to reduce the native silver oxide at the $\mathrm{Ag} / \mathrm{TiO}_{2}$ interface. The use of nearly-monodisperse plasmonic nanoparticles allows us to detect surface chemical modifications down to a single atomic layer and measure sub-nm variations in their shell thickness. A detailed understanding of the equilibration of photogenerated charge carriers in metal/semiconductor heterostructures is of paramount importance for the development of efficient energy conversion materials and for the design of novel plasmonic sensors for chemical and physical processes.

\section{ASSOCIATED CONTENT}

\section{Supporting Information.}

The Supporting Information is available free of charge on the ACS Publications website.

Supplementary information on 1) Experimental setup for UV irradiation, 2) Effect of silver oxide on the LSPR position of Ag nanoparticle, 3) $\mathrm{Ag} @ \mathrm{TiO}_{2} \mathrm{LSPR}$ recovery after exposure to air, 4) Dielectric function of sol-gel $\left.\mathrm{TiO}_{2}, 5\right)$ Effect of charge density increase on the LSPR of $\mathrm{Ag} @ \mathrm{TiO}_{2}$ nanoparticles, 6) Ag and Au spectral sensitivity to the surrounding environment, 7) Electron-beam induced shrinking of $\mathrm{TiO}_{2}$, 8) LSPR blueshift for $\mathrm{Ag} @ \mathrm{TiO}_{2}$ and $\mathrm{Au} @ \mathrm{TiO}_{2}$ due to electron accumulation in $\mathrm{TiO}_{2}$, 9) Effect of CID on extinction spectra of $\mathrm{Ag} @ \mathrm{TiO}_{2}$ nanoparticles. (PDF)

\section{AUTHOR INFORMATION}

\section{Corresponding Author}

*a.baldi@differ.nl

\section{ACKNOWLEDGMENT}

We gratefully acknowledge Erwin Zoethout for the ellipsometry measurements as well as Ruben Hamans, Rifat Kamarudheen, Gayatri Kumari, Erik Garnett, Sven Askes, and Jenny Kontoleta for insightful discussions.
This work is part of the research programme of the Foundation for Fundamental Research on Matter (FOM), which is financially supported by the Netherlands Organisation for Scientific Research (NWO).

\section{REFERENCES}

(1) Bohren, C. F.; Huffman, D. R. Absorption and Scattering of Light by Small Particles; Wiley-VCH: New York, 1983.

(2) Mie, G. Beitrage Zur Optik Truber Medien, Speziell Kolloidaler Metallosungen. Ann. Phys. 1908, 330, 377-445.

(3) Anker, J. N.; Hall, W. P.; Lyandres, O.; Shah, N. C.; Zhao, J.; Van Duyne, R. P. Biosensing with Plasmonic Nanosensors. Nat. Mater. 2008, 7, 442-453.

(4) Mayer, K. M.; Hafner, J. H. Localized Surface Plasmon Resonance Sensors. Chem. Rev. 2011, 111, 3828-3857.

(5) Saha, K.; Agasti, S.; Kim, C.; Li, X.; Rotello, V. Gold Nanoparticles in Chemical and Biological Sensing. Chem. Rev. 2012, 112, 2739-2779.

(6) Langer, J.; Novikov, S. M.; Liz-Marzán, L. M. Sensing Using Plasmonic Nanostructures and Nanoparticles. Nanotechnology 2015, $26,1-28$.

(7) Larsson, E. M.; Langhammer, C.; Zorić, I.; Kasemo, B. Nanoplasmonic Probes of Catalytic Reactions. Science (80-. ). 2009, 326, 1091-1094.

(8) Kreno, L. E.; Hupp, J. T.; Van Duyne, R. P. Metal-Organic Framework Thin Film for Enhanced Localized Surface Plasmon Resonance Gas Sensing. Anal. Chem. 2010, 82, 8042-8046.

(9) Liu, N.; Tang, M. L.; Hentschel, M.; Giessen, H.; Alivisatos, A. P. Nanoantenna-Enhanced Gas Sensing in a Single Tailored Nanofocus. Nat. Mater. 2011, 10, 631-636.

(10) Sönnichsen, C.; Reinhard, B. M.; Liphardt, J.; Alivisatos, A. P. A Molecular Ruler Based on Plasmon Coupling of Single Gold and Silver Nanoparticles. Nat. Biotechnol. 2005, 23, 741-745.

(11) Hall, W. P.; Anker, J. N.; Lin, Y.; Modica, J.; Mrksich, M.; Van Duyne, R. P. A Calcium-Modulated Plasmonic Switch. J. Am. Chem. Soc. 2008, 130, 5836-5837.

(12) Ament, I.; Prasad, J.; Henkel, A.; Schmachtel, S.; Sönnichsen, C. Single Unlabeled Protein Detection on Individual Plasmonic Nanoparticles. Nano Lett. 2012, 12, 1092-1095.

(13) Novo, C.; Funston, A. M.; Gooding, A. K.; Mulvaney, P. Electrochemical Charging of Single Gold Nanorods Carolina. J. Am. Chem. Soc. Comms. 2009, 131, 14664-14666.

(14) Mulvaney, P.; Pérez-Juste, J.; Giersig, M.; Liz-Marzán, L. M.; Pecharromán, C. Drastic Surface Plasmon Mode Shifts in Gold Nanorods due to Electron Charging. Plasmonics 2006, 1, 61-66.

(15) Novo, C.; Funston, A. M.; Mulvaney, P. Direct Observation of Chemical Reactions on Single Gold Nanocrystals Using Surface Plasmon Spectroscopy. Nat. Nanotechnol. 2008, 3, 598-602.

(16) Amirav, L.; Alivisatos, A. Photocatalytic Hydrogen Production with Tunable Nanorod Heterostructures. J. Phys. Chem. Lett. 2010, 1, 1051-1054.

(17) Yoo, H.; Bae, C.; Yang, Y.; Lee, S.; Kim, M.; Kim, H.; Kim, Y.; Shin, H. Spatial Charge Separation in Asymmetric Structure of $\mathrm{Au}$ Nanoparticle on $\mathrm{TiO}_{2}$ Nanotube by Light-Induced Surface Potential Imaging. Nano Lett. 2014, 14, 4413-4417.

(18) Kamat, P. V. Manipulation of Charge Transfer Across Semiconductor Interface. J. Phys. Chem. Lett. 2012, 3, 663-672.

(19) Subramanian, V.; Wolf, E. E.; Kamat, P. V. Catalysis with $\mathrm{TiO}_{2} /$ Gold Nanocomposites. Effect of Metal Particle Size on the Fermi Level Equilibration. J. Am. Chem. Soc. 2004, 126, 4943-4950.

Hirakawa, T.; Kamat, P. V. Photoinduced Electron Storage and Surface Plasmon Modulation in $\mathrm{Ag} @ \mathrm{TiO}_{2}$ Clusters. Langmuir 2004, 20, 5645-5647.

(21) Hirakawa, T.; Kamat, P. V. Charge Separation and Catalytic Activity of $\mathrm{Ag} @ \mathrm{TiO}_{2}$ Core-Shell Composite Clusters under UV-Irradiation. J. Am. Chem. Soc. 2005, 127, 3928-3934.

(22) Takai, A.; Kamat, P. V. Capture, Store, and Discharge. Shuttling Photogenerated Electrons across $\mathrm{TiO}_{2}$-Silver Interface. ACS Nano 2011, 5, 7369-7376.

(23) Sotelo-Vazquez, C.; Ben-jaber, S.; Peveler, W. J.; Quesada-cabrera, 
R.; Corte, E.; Abdul-karim, N.; Maier, S. A.; Parkin, I. P. PhotoInduced Enhanced Raman Spectroscopy for Universal Ultra-Trace Detection of Explosives, Pollutants and Biomolecules. Nat. Commun. 2016, 7, 12189.

(24) Navarrete, J.; Siefe, C.; Alcantar, S.; Belt, M.; Stucky, G. D.; Moskovits, M. Merely Measuring the UV - Visible Spectrum of Gold Nanoparticles Can Change Their Charge State. Nano Lett. 2018, 18, 3-8.

(25) Brown, A. M.; Sheldon, M. T.; Atwater, H. A. Electrochemical Tuning of the Dielectric Function of $\mathrm{Au}$ Nanoparticles. ACS Photonics 2015, 2, 459-464.

(26) Awazu, K.; Fujimaki, M.; Rockstuhl, C.; Tominaga, J.; Murakami, H.; Ohki, Y.; Yoshida, N.; Watanabe, T. A Plasmonic Photocatalyst Consisting of Silver Nanoparticles Embedded in Titanium Dioxide. J. Am. Chem. Soc. 2008, 130, 1676-1680.

(27) Zijlstra, P.; Paulo, P. M. R.; Yu, K.; Xu, Q. H.; Orrit, M. Chemical Interface Damping in Single Gold Nanorods and Its near Elimination by Tip-Specific Functionalization. Angew. Chemie - Int. Ed. 2012, 51, 8352-8355.

(28) Foerster, B.; Joplin, A.; Kaefer, K.; Celiksoy, S.; Link, S.; Sönnichsen, C. Chemical Interface Damping Depends on Electrons Reaching the Surface. ACS Nano 2017, 11, 2886-2893.

(29) Dadosh, T. Synthesis of Uniform Silver Nanoparticles with a Controllable Size. Mater. Lett. 2009, 63, 2236-2238.

(30) Neus, B.; Comenge, J.; Puntes, V. Kinetically Controlled Seeded Growth Synthesis of Citrate-Stabilized Gold Nanoparticles of up to 200 Nm: Size Focusing versus Ostwald Ripening. Langmuir 2011, 11098-11105.

(31) Goebl, J.; Joo, J. B.; Dahl, M.; Yin, Y. Synthesis of Tailored Au@TiO Core-Shell Nanoparticles for Photocatalytic Reforming of Ethanol. Catal. Today 2014, 225, 90-95.

(32) Lee, J. W.; Othman, M. R.; Eom, Y.; Lee, T. G.; Kim, W. S.; Kim, J. The Effects of Sonification and $\mathrm{TiO}_{2}$ Deposition on the MicroCharacteristics of the Thermally Treated $\mathrm{SiO}_{2} / \mathrm{TiO}_{2}$ Spherical Coreshell Particles for Photo-Catalysis of Methyl Orange. Microporous Mesoporous Mater. 2008, 116, 561-568.

(33) Yin, Y.; Li, Z.-Y.; Zhong, Z.; Gates, B.; Xia, Y.; Venkateswaran, S. Synthesis and Characterization of Stable Aqueous Dispersions of Silver Nanoparticles through the Tollens Process. J. Mater. Chem. 2002, 12, 522-527.

(34) Yang, W. Improved Recursive Algorithm for Light Scattering by a Multilayered Sphere. Appl. Opt. 2003, 42, 1710-1720.

(35) Suzuki, T. X-Ray Study on the Binding Properties of $\mathrm{Cu}_{2} \mathrm{O}$ and $\mathrm{Ag}_{2} \mathrm{O}$ Crystals. J. Phys. Soc. Japan 1960, 15, 2018-2024.

(36) Alves, L.; Boronat, M.; Corma, A.; Correa-duarte, M. A. Synthesis and Stabilization of Subnanometric Gold Oxide Nanoparticles. 2011, $2-6$.

(37) Johnson, P. B.; Christy, R. W. Optical Constants of the Noble Metals. Phys. Rev. B 1972, 6, 4370-4379.

(38) Ehrenreich, H.; Philipp, H. R. Optical Properties of Ag and Cu. Phys Rev. 1962, 128, 1622-1629.

(39) Scanlon, M. D.; Peljo, P.; Méndez, M. A.; Smirnov, E.; Girault, H. H. Charging and Discharging at the Nanoscale: Fermi Level Equilibration of Metallic Nanoparticles. Chem. Sci. 2015, 6, 27052720.

(40) Alam, M. J.; Cameron, D. C. Preparation and Characterization of TiO2 Thin Films by Sol-Gel Method. J. Sol-Gel Sci. Technol. 2002, $137-145$.

(41) Shin, H.; DeGuire, M. R.; Heuer, A. H. Electrical Properties of TiO2 Thin Films Formed on Self-Assembled Organic Monolayers on Silicon. J. Appl. Phys. 1998, 83, 3311-3317.

(42) Wyman, J. The Dielectric Constant of Mixtures of Ethyl Alcohol and Water from -5 to $40^{\circ}$. J. Am. Chem. Soc. 1931, 53, 3292-3301.

(43) Dweydari, A. W.; Mee, C. H. B. Work Function Measurements on
(100) and (110) Surfaces of Silver. Phys. Status Solidi 1975, 27, 223 230.

(44) Kandel, H.; Lu, J.; Jiang, J.; Chen, P.; Matras, M.; Craig, N.; Trociewitz, U. P.; Hellstrom, E. E.; Larbalestier, D. C. Development of $\mathrm{TiO} 2$ Electrical Insulation Coating on Ag-Alloy Sheathed $\mathrm{Bi} 2 \mathrm{Sr}_{2} \mathrm{CaCu}_{2} \mathrm{O}_{8-\mathrm{x}}$ Round-Wire. Supercond. Sci. Technol. 2015, 28, 35010 .

(45) D. Bahnemann, A. Henglein, J. Lilie, and L. S. Flash Photolysis Observation of the Absorption Spectra of Trapped Positive Holes and Electrons in Colloidal Titanium Dioxide. J. Phys. Chem. 1984, 88, 709-711.

(46) Bahnemann, Detlef W.; Hilgendorff, Marcus; Memming, R. Charge Carrier Dynamics at $\mathrm{TiO}_{2}$ Particles: Reactivity of Free and Trapped Holes. J. Phys. Chem. B 1997, 101, 4265-4275.

(47) Schneider, J.; Matsuoka, M.; Takeuchi, M.; Zhang, J.; Horiuchi, Y.; Anpo, M.; Bahnemann, D. W. Understanding $\mathrm{TiO}_{2}$ Photocatalysis: Mechanisms and Materials. Chem. Rev. 2014, 114, 9919-9986.

(48) Yu, J. C.; Yu, J.; Ho, W.; Zhao, J. Light-Induced Super-Hydrophilicity and Photocatalytic Activity of Mesoporous $\mathrm{TiO}_{2}$ Thin Films. J. Photochem. Photobiol. A Chem. 2002, 148, 331-339.

(49) Gao, Y.; Masuda, Y.; Koumoto, K. Light-Excited Superhydrophilicity of Amorphous $\mathrm{TiO}_{2}$ Thin Films Deposited in an Aqueous Peroxotitanate Solution. Langmuir 2004, 20, 3188-3194.

(50) Karuppuchamy, S.; Jeong, J. M. Super-Hydrophilic Amorphous Titanium Dioxide Thin Film Deposited by Cathodic Electrodeposition. Mater. Chem. Phys. 2005, 93, 251-254.

(51) Brinker, C. J.; Sehgal, R.; Hietala, S. L.; Deshpande, R.; Smith, D. M.; Loy, D.; Ashley, C. S. Sol-Gel Strategies for Controlled Porosity Inorganic Materials. J. Memb. Sci. 1994, 94, 85-102.

(52) Guo, B.; Liu, Z.; Hong, L.; Jiang, H. Sol Gel Derived Photocatalytic Porous TiO2thin Films. Surf. Coatings Technol. 2005, 198, 24-29.

(53) Liu, Y.; Daum, P. H. Relationship of Refractive Index to Mass Density and Self-Consistency of Mixing Rules for Multicomponent Mixtures like Ambient Aerosols. J. Aerosol Sci. 2008, 39, 974-986.

(54) Ottermann, C. . R.; Bange, K. Correlation between the Density of $\mathrm{TiO}_{2}$ Films and Their Properties. Thin Solid Films 1996, 286, 32-34.

(55) Mergel, D.; Buschendorf, D.; Eggert, S.; Grammes, R.; Samset, B. Density and Refractive Index of $\mathrm{TiO}_{2}$ Films Prepared by Reactive Evaporation. Thin Solid Films 2000, 371, 218-224.

(56) Matthias, A.; Raićevic, N.; Donfeu Tchana, R.; Kip, D.; Deubener, J. Density Dependence of Refractive Index of Nanoparticle-Derived Titania Films on Glass. Thin Solid Films 2014, 558, 86-92.

(57) Bilmes, S. A.; Mandelbaum, P.; Alvarez, F.; Victoria, N. M. Surface and Electronic Structure of Titanium Dioxide Photocatalysts. J. Phys. Chem. B 2000, 104, 9851-9858.

(58) Persson, B. N. J. Polarizability of Small Spherical Metal Particles: Influence of the Matrix Environment. Surf. Sci. 1993, 281, 153-162.

(59) Hartland, G. V. Optical Studies of Dynamics in Noble Metal Nanostructures. Chem. Rev. 2011, 111, 3858-3887.

(60) Linic, S.; Aslam, U.; Boerigter, C.; Morabito, M. Photochemical Transformations on Plasmonic Metal Nanoparticles. Nat. Mater. 2015, 14, 567-576.

(61) Wu, K.; Chen, J.; Mcbride, J. R.; Lian, T. Efficient Hot-Electron Transfer by a Plasmon-Induced Interfacial Charge-Transfer Transition. Science (80-. ). 2015, 349, 632.

(62) Bosbach, J.; Hendrich, C.; Stietz, F.; Vartanyan, T.; Träger, F. Ultrafast Dephasing of Surface Plasmon Excitation in Silver Nanoparticles: Influence of Particle Size, Shape, and Chemical Surrounding. Phys. Rev. Lett. 2002, 89, 2-5.

(63) Clavero, C. Plasmon-Induced Hot-Electron Generation at Nanoparticle/metal-Oxide Interfaces for Photovoltaic and Photocatalytic Devices. Nat. Photonics 2014, 8, 95-103. 

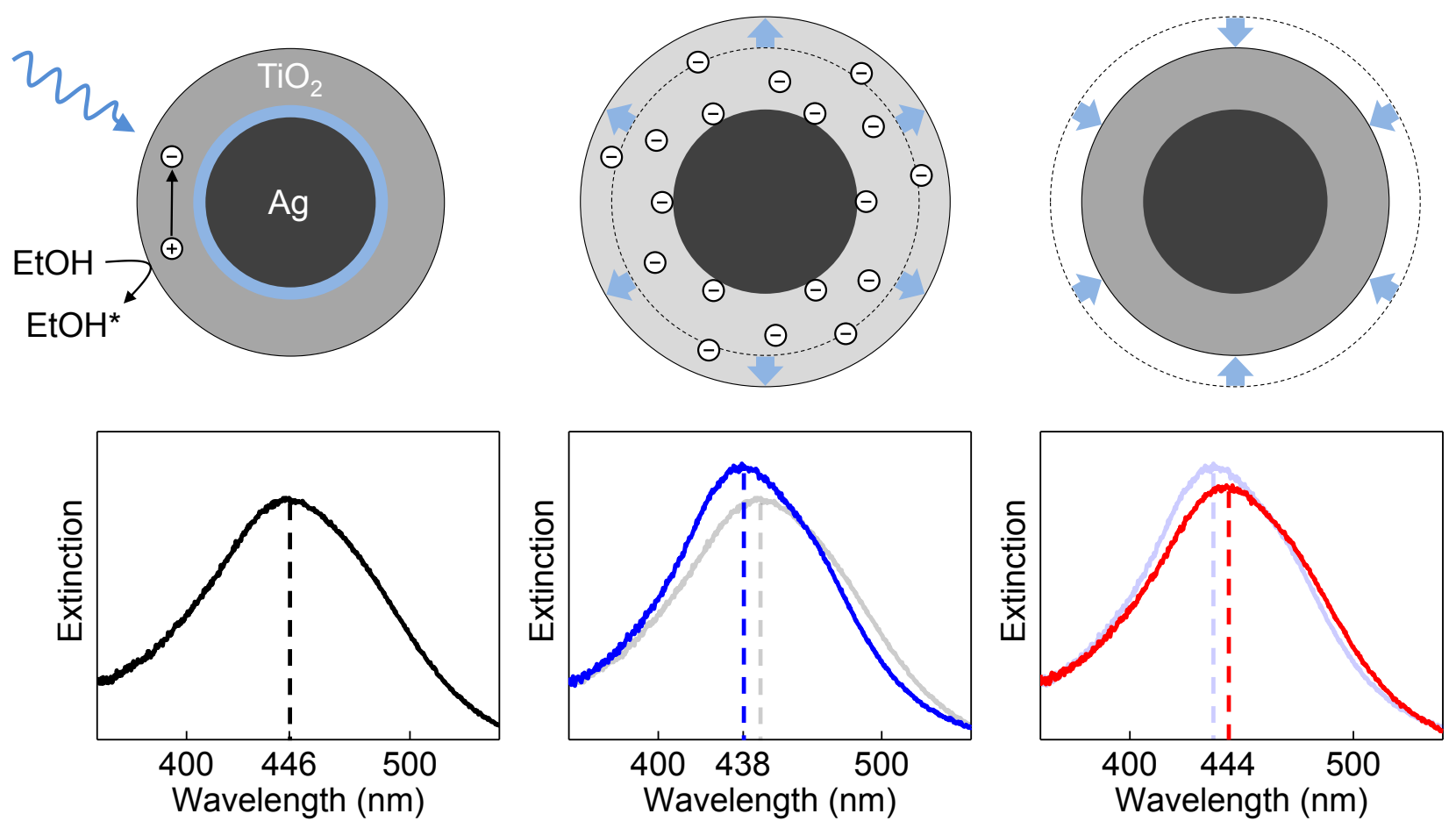